J. Korean Math. Soc. 50 (2013), No. 4, pp. 829-845

http://dx.doi.org/10.4134/JKMS.2013.50.4.829

\title{
A NEW 3-PARAMETER CURVATURE CONDITION PRESERVED BY RICCI FLOW
}

\author{
XIANG GaO
}

\begin{abstract}
In this paper, we firstly establish a family of curvature invariant conditions lying between the well-known 2-nonnegative curvature operator and nonnegative curvature operator along the Ricci flow. These conditions are defined by a set of inequalities involving the first four eigenvalues of the curvature operator, which are named as 3-parameter $\lambda$-nonnegative curvature conditions. Then a related rigidity property of manifolds with 3-parameter $\lambda$-nonnegative curvature operators is also derived. Based on these, we also obtain a strong maximum principle for the 3 -parameter $\lambda$-nonnegativity along Ricci flow.
\end{abstract}

\section{Introduction and main results}

One of the basic problems in Riemannian geometry is to relate curvature and topology. In [1], Böhm and Wilking prove that $n$-dimensional closed Riemannian manifolds with 2-positive curvature operators are diffeomorphic to spherical space forms, i.e., they admit metrics with constant positive sectional curvature. One of the key points of their theorem is that the 2-positive or 2-nonnegative curvature condition is preserved by the Ricci flow.

Recall that the Riemannian curvature tensor is defined by

$$
R(X, Y) Z=\nabla_{X} \nabla_{Y} Z-\nabla_{Y} \nabla_{X} Z-\nabla_{[X, Y]} Z
$$

for tangent vectors $X, Y$ and $Z$. The Riemannian curvature operator, denoted by $\mathcal{R}$, is the symmetric bilinear form on $\wedge^{2} T M^{n}$ (or self-adjoint transformation of $\wedge^{2} T M^{n}$ ) defined by

$$
\mathcal{R}(X \wedge Y, Z \wedge W)=\langle\mathcal{R}(X \wedge Y), Z \wedge W\rangle=2\langle R(X, Y) W, Z\rangle
$$

for tangent vectors $X, Y, Z$ and $W$.

Let $\left\{\mu_{\alpha}(\mathcal{R}) \mid \mu_{1}(\mathcal{R}) \leq \cdots \leq \mu_{N}(\mathcal{R})\right\}_{\alpha=1}^{N}$, where $N=n(n-1) / 2$, denote the eigenvalues of the curvature operator $\mathcal{R}$. We have the following definition

Received September 9, 2011; Revised February 7, 2013.

2010 Mathematics Subject Classification. 58G25, 35P05.

Key words and phrases. Ricci flow, 3-parameter $\lambda$-nonnegative curvature operator, maximum principle.

This work is supported by the Fundamental Research Funds for the Central Universities and NSFC11101267. 
which was studied by Chen in dimension 4 [2] and mentioned in the work of Micallef and Moore [7]:

Definition 1.1 (2-positive curvature operator). A Riemannian manifold $\left(M^{n}, g\right)$ has 2-positive curvature operator if

$$
\mu_{\alpha}(\mathcal{R})+\mu_{\beta}(\mathcal{R})>0
$$

for arbitrary $\alpha \neq \beta$.

Remark 1 . The 2-nonnegative curvature operator is defined in the obvious way with $\geq$ replacing $>$ in $(1)$

In this paper, we deal with a generalization of the 2-nonnegative curvature operator, which is named as 3 -parameter $\left(\lambda_{1}, \lambda_{2}, \lambda_{3}\right)$-nonnegative curvature operator. It relies on four eigenvalues of the Riemannian curvature operator $\mathcal{R}$.

On a Riemannian manifold $M^{n}$, let $\left\{\omega_{\alpha}\right\}_{\alpha=1}^{N}$ be an orthonormal basis of eigenvectors of $\mathcal{R}$ in $\mathfrak{s o}(n, \mathbb{R})$ with corresponding eigenvalues $\mu_{1}(\mathcal{R}) \leq \mu_{2}(\mathcal{R}) \leq$ $\cdots \leq \mu_{N}(\mathcal{R})$, where $N=n(n-1) / 2$, and let

$$
\Lambda=\left\{\begin{array}{l|l}
\left(x_{1}, x_{2}, x_{3}\right) \in[0,1] \times[0,1] \times[0,1] \mid \begin{array}{c}
x_{3} \leq x_{2} \leq x_{1} \\
0<1-\left(x_{i}+x_{j}\right) x_{j} \leq x_{i} \leq 1, \\
x_{i}+x_{j} \geq 1,1 \leq i \neq j \leq 3
\end{array}
\end{array}\right\} .
$$

Then as Definition 1.1, the definition of 3-parameter $\left(\lambda_{1}, \lambda_{2}, \lambda_{3}\right)$-nonnegative curvature operator is given as follows:

Definition 1.2 (3-parameter $\left(\lambda_{1}, \lambda_{2}, \lambda_{3}\right)$-convex cone). Let $\left\{\omega_{\alpha}\right\}_{\alpha=1}^{N}$ be an orthonormal basis for the vector space $S_{B}^{2}\left(\wedge^{2} \mathbb{R}^{n}\right)$ of algebraic curvature operators, and let $C_{\lambda_{1}, \lambda_{2}, \lambda_{3}}$ be the set of all $\mathcal{R}$ 's satisfying

(3)

$$
\begin{aligned}
& C_{\lambda_{1}, \lambda_{2}, \lambda_{3}}
\end{aligned}
$$

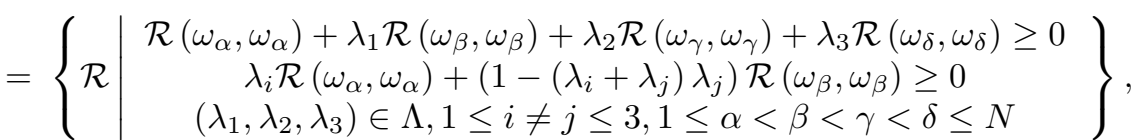

Then $C_{\lambda_{1}, \lambda_{2}, \lambda_{3}}$ can be embedded in each fiber of the fiber bundle $S_{B}^{2}\left(\wedge^{2} T M^{n}\right)$ of curvature operators on $M^{n}$ because of the $O(n)$-invariance. Then we call $C_{\lambda_{1}, \lambda_{2}, \lambda_{3}}$ a 3-parameter $\left(\lambda_{1}, \lambda_{2}, \lambda_{3}\right)$-convex cone.

Definition 1.3 (3-parameter $\left(\lambda_{1}, \lambda_{2}, \lambda_{3}\right)$-nonnegative curvature operator). If for each $x \in M^{n}$, the curvature operator $\mathcal{R}$ at x belongs to $C_{\lambda_{1}, \lambda_{2}, \lambda_{3}}$, then we say the Riemannian manifold $\left(M^{n}, g\right)$ has $\left(\lambda_{1}, \lambda_{2}, \lambda_{3}\right)$-nonnegative curvature operator.

Remark 2. The 3-parameter $\left(\lambda_{1}, \lambda_{2}, \lambda_{3}\right)$-positive curvature operator $C_{\lambda_{1}, \lambda_{2}, \lambda_{3}}^{+}$ is defined in the obvious way with $>$ replacing $\geq$ in (3). 
Remark 3. It is easy to see that when $\lambda_{1}=1, \lambda_{2}=\lambda_{3}=0$, the 3 -parameter $(1,0,0)$-nonnegative curvature turns into 2 -nonnegative curvature operator in [1]. Actually, it can be seen in Section 2 that

$$
\bigcap_{\left(\lambda_{1}, \lambda_{2}, \lambda_{3}\right) \in \Lambda} C_{\lambda_{1}, \lambda_{2}, \lambda_{3}}=\left\{\mathcal{R} \mid \mathcal{R}_{\alpha \alpha} \geq 0,1 \leq \alpha \leq N\right\}
$$

and

$$
\bigcup_{\left(\lambda_{1}, \lambda_{2}, \lambda_{3}\right) \in \Lambda} C_{\lambda_{1}, \lambda_{2}, \lambda_{3}}=\left\{\mathcal{R} \mid \mathcal{R}_{\alpha \alpha}+\mathcal{R}_{\beta \beta} \geq 0,1 \leq \alpha<\beta \leq N\right\} .
$$

Remark 4. But the 3-parameter $\left(\lambda_{1}, \lambda_{2}, \lambda_{3}\right)$-nonnegative curvature operator is not always equal to 2-nonnegative curvature. For example, when

$$
\left(\lambda_{1}, \lambda_{2}, \lambda_{3}\right)=\left(\frac{3}{4}, \frac{1}{2}, 0\right)
$$

or more generally, when $\lambda_{1}>1-\left(\lambda_{1}+\lambda_{2}\right) \lambda_{2}$ and $\lambda_{3}=0$, the curvature operator $\mathcal{R}_{11}=-1, \mathcal{R}_{22}=1, \ldots$ which satisfies 2 -nonnegativity is not 3 -parameter nonnegative.

Setting $\lambda=\left(\lambda_{1}, \lambda_{2}, \lambda_{3}\right) \in \Lambda$, we will use 3 -parameter $\lambda$-nonnegative curvature operator to represent the 3 -parameter $\left(\lambda_{1}, \lambda_{2}, \lambda_{3}\right)$-nonnegativity in the rest of paper, which would lighten a bit the notations. Now, we formulate one of the main results of this paper as follows:

Theorem 1.4. The convex cone $C_{\lambda_{1}, \lambda_{2}, \lambda_{3}}$ of 3-parameter $\lambda$-nonnegative algebraic curvature operators is preserved as a subset of $S_{B}^{2}\left(\wedge^{2} \mathbb{R}^{n}\right)$ by Hamilton's ODE

$$
\frac{d \mathcal{R}}{d t}=\mathcal{R}^{2}+\mathcal{R}^{\#}
$$

On the other hand, the following maximum principle established by Hamilton, Chow and Lu (see [4]) is very useful in the research of Ricci flow:

Theorem 1.5 (Maximum principle for convex sets). Let $\left(M^{n}, g(t)\right)$ be a solution to the Ricci flow and let $K(t) \subset E=\wedge^{2} T M^{n} \otimes_{S} \wedge^{2} T M^{n}$ be subsets which are invariant under parallel translation and whose intersections $K(t)_{x}=K(t) \cap E_{x}$ with each fiber are closed and convex. Suppose also that the set $\{(v, t) \in E \times[0, T) \mid v \in K(t)\}$ is closed in $E \times[0, T)$ and suppose the ODE

$$
\frac{d M}{d t}=M^{2}+M^{\#}
$$

has the property that for any $M\left(t_{0}\right) \in K\left(t_{0}\right)$, we have $M(t) \in K(t)$ for arbitrary $t \in\left[t_{0}, T\right)$. Then, if $\mathcal{R}(0) \in K(0)$, we have $\mathcal{R}(t) \in K(t)$ for arbitrary $t \in[0, T)$.

Using Theorem 1.4 and Theorem 1.5, we can derive directly the weak maximum principle for 3 -parameter $\lambda$-nonnegativity: 
Theorem 1.6 (Weak maximum principle for 3-parameter $\lambda$-nonnegativity). Let $\left(M^{n}, g(t)\right), t \in[0, T)$ be a solution to the Ricci flow on a closed manifold. If the curvature operator $\mathcal{R}(g(0))$ is 3-parameter $\lambda$-nonnegative, then for any $0 \leq t<T$, the curvature operator $\mathcal{R}(g(t))$ is also 3-parameter $\lambda$-nonnegative.

Remark 5. Since when $\lambda_{1}=1, \lambda_{2}=\lambda_{3}=0$, the 3 -parameter $(1,0,0)$ nonnegative curvature operator turns into the well-known 2-nonnegative curvature operator studied by Chen in dimension 4 [2] and mentioned in the work of Micallef and Moore [7], as a corollary of Theorem 1.6, we obtain the invariance of 2-nonnegative curvature along the Ricci flow again.

Theorem 1.7 (Weak maximum principle for 2-nonnegativity). Let $\left(M^{n}, g(t)\right)$, $t \in[0, T)$ be a solution to the Ricci flow on a closed manifold. If the curvature operator $\mathcal{R}(g(0))$ is 2-nonnegative, then for any $0 \leq t<T$, the curvature operator $\mathcal{R}(g(t))$ is also 2-nonnegative.

On the other hand, in [1] Böhm and Wilking also derive a convergence result for the 2-positive curvature operator along the Ricci flow:

Theorem 1.8 (Böhm and Wilking). On a compact manifold, the normalized Ricci flow evolves a Riemannian metric with 2-positive curvature operator to a limit metric with constant sectional curvature.

Thus by using Theorem 1.8 and the result

$$
\bigcup_{\left(\lambda_{1}, \lambda_{2}, \lambda_{3}\right) \in \Lambda} C_{\lambda_{1}, \lambda_{2}, \lambda_{3}}^{+}=\left\{\mathcal{R} \mid \mathcal{R}_{\alpha \alpha}+\mathcal{R}_{\beta \beta}>0,1 \leq \alpha<\beta \leq N\right\},
$$

which is proved in Section 2, we can derive a similar convergence result of 3 -parameter $\lambda$-positive curvature operator along the Ricci flow as follows:

Theorem 1.9. For arbitrary $\lambda=\left(\lambda_{1}, \lambda_{2}, \lambda_{3}\right) \in \Lambda$, on a compact manifold, the normalized Ricci flow

$$
\frac{\partial g}{\partial t}=-2 R i c(g)+\frac{2}{n} r g
$$

evolves a Riemannian metric with 3-parameter $\lambda$-positive curvature operator to a limit metric with constant sectional curvature.

The paper is organized as follows. In Section 2, we present some preliminaries and obtain a rigidity property of manifolds with 3 -parameter $\lambda$-nonnegative curvature operators. In Section 3 , we prove Theorem 1.4 by a direct calculation. In Section 4, we prove the strong maximum principle for the 3 -parameter $\lambda$-nonnegativity along the Ricci flow.

\section{Preliminaries and a rigidity property}

Let $(V,\langle\rangle$,$) be a Euclidean vector space. Then \wedge^{2} V$ has a natural scalar product defined by

$$
\left\langle u \wedge v, u^{\prime} \wedge v^{\prime}\right\rangle=\left\langle u, u^{\prime}\right\rangle\left\langle v, v^{\prime}\right\rangle-\left\langle u, v^{\prime}\right\rangle\left\langle u^{\prime}, v\right\rangle .
$$


One can then identify $\wedge^{2} V$ with $\mathfrak{s o}(V)$ by the following natural isomorphism:

$$
\begin{gathered}
\wedge^{2} V \rightarrow \mathfrak{s o}(V) \\
u \wedge v \mapsto(x \mapsto\langle u, x\rangle v-\langle v, x\rangle u) .
\end{gathered}
$$

Moreover, this isomorphism is an isometry when $\mathfrak{s o}(V)$ is endowed with the scalar product $\langle A, B\rangle=\frac{1}{2} \operatorname{Tr}\left(A B^{T}\right)$. The Lie algebra structure on $\wedge^{2} V$ that allows one to define the \# operator (see below) on curvature operators comes from this identification and the usual Lie bracket on $\mathfrak{s o}(V)$ (namely, the commutator of endomorphisms).

In this paper, we set the Euclidean vector space $V=\wedge^{2} T^{*} M^{n}$. For an orthonormal basis $\left\{\varphi^{\alpha}\right\}_{\alpha=1}^{N}$ of $\wedge^{2} T^{*} M^{n}$, the structure constants for the Lie bracket are given by

$$
\left[\varphi^{\alpha}, \varphi^{\beta}\right]=\sum_{\gamma} c_{\gamma}^{\alpha \beta} \varphi^{\gamma}
$$

Hence

$$
c_{\gamma}^{\alpha \beta}=\left\langle\left[\varphi^{\alpha}, \varphi^{\beta}\right], \varphi^{\gamma}\right\rangle
$$

and $c_{\gamma}^{\alpha \beta}$ is anti-symmetric in all 3 variables. The sharp product operator \# for the Lie algebra $\wedge^{2} T^{*} M^{n}$ with the dual orthonormal basis $\left\{\varphi_{\alpha}\right\}_{\alpha=1}^{N}$ for $\wedge^{2} T M^{n}$ is defined by

$$
(A \# B)_{\alpha \beta}=(A \# B)\left(\varphi_{\alpha}, \varphi_{\beta}\right)=\frac{1}{2} c_{\alpha}^{\gamma \eta} c_{\beta}^{\delta \theta} A_{\gamma \delta} B_{\eta \theta}
$$

For the 3-parameter $\lambda$-nonnegative curvature operator convex cone $C_{\lambda_{1}, \lambda_{2}, \lambda_{3}}$, we have the following interesting property:

Theorem 2.1. Let

$$
\Lambda=\left\{\begin{array}{l|l}
\left(x_{1}, x_{2}, x_{3}\right) \in[0,1] \times[0,1] \times[0,1] & \begin{array}{c}
x_{3} \leq x_{2} \leq x_{1} \\
0<1-\left(x_{i}+x_{j}\right) x_{j} \leq x_{i} \leq 1 \\
x_{i}+x_{j} \geq 1,1 \leq i \neq j \leq 3
\end{array}
\end{array}\right\}
$$

Then we have

$$
\bigcup_{\left(\lambda_{1}, \lambda_{2}, \lambda_{3}\right) \in \Lambda} C_{\lambda_{1}, \lambda_{2}, \lambda_{3}}=\left\{\mathcal{R} \mid \mathcal{R}_{\alpha \alpha}+\mathcal{R}_{\beta \beta} \geq 0,1 \leq \alpha<\beta \leq N\right\}
$$

and

$$
\bigcup_{\left(\lambda_{1}, \lambda_{2}, \lambda_{3}\right) \in \Lambda} C_{\lambda_{1}, \lambda_{2}, \lambda_{3}}^{+}=\left\{\mathcal{R} \mid \mathcal{R}_{\alpha \alpha}+\mathcal{R}_{\beta \beta}>0,1 \leq \alpha<\beta \leq N\right\} .
$$

Proof. First we prove (4). For any $\mathcal{R} \in\left\{\mathcal{R} \mid \mathcal{R}_{\alpha \alpha} \geq 0,1 \leq \alpha \leq N\right\}$, we have

$$
\mathcal{R}_{\alpha \alpha}+\lambda_{1} \mathcal{R}_{\beta \beta}+\lambda_{2} \mathcal{R}_{\gamma \gamma}+\lambda_{3} \mathcal{R}_{\delta \delta} \geq 0
$$

and

$$
\lambda_{i} \mathcal{R}_{\alpha \alpha}+\left(1-\left(\lambda_{i}+\lambda_{j}\right) \lambda_{j}\right) \mathcal{R}_{\beta \beta} \geq 0
$$


for arbitrary $1 \leq \alpha<\beta<\gamma<\delta \leq N, 1 \leq i \neq j \leq 3$ and $\left(\lambda_{1}, \lambda_{2}, \lambda_{3}\right) \in \Lambda$. This implies $\mathcal{R} \in C_{\lambda_{1}, \lambda_{2}, \lambda_{3}}$. Then

$$
\left\{\mathcal{R} \mid \mathcal{R}_{\alpha \alpha} \geq 0,1 \leq \alpha \leq N\right\} \subset C_{\lambda_{1}, \lambda_{2}, \lambda_{3}}
$$

for arbitrary $\left(\lambda_{1}, \lambda_{2}, \lambda_{3}\right) \in \Lambda$, and hence

$$
\bigcap_{\left(\lambda_{1}, \lambda_{2}, \lambda_{3}\right) \in \Lambda} C_{\lambda_{1}, \lambda_{2}, \lambda_{3}} \supset\left\{\mathcal{R} \mid \mathcal{R}_{\alpha \alpha} \geq 0,1 \leq \alpha \leq N\right\} .
$$

Conversely, without loss of generality, we consider a fixed $\bar{\lambda}_{1}$. Since

$$
0 \leq \frac{\sqrt{\bar{\lambda}_{1}^{2}+4}-\bar{\lambda}_{1}}{2}=\frac{2}{\sqrt{\bar{\lambda}_{1}^{2}+4}+\bar{\lambda}_{1}} \leq 1
$$

and

$$
\frac{2}{\sqrt{\bar{\lambda}_{1}^{2}+4}+\bar{\lambda}_{1}}<\bar{\lambda}_{1}
$$

for sufficiently large $\bar{\lambda}_{1}$, we have $\left(\overline{\lambda_{1}}, \lambda_{2}, \lambda_{3}\right) \in \Lambda$ if

$$
\left|\lambda_{i}-\frac{\sqrt{\bar{\lambda}_{1}^{2}+4}-\bar{\lambda}_{1}}{2}\right|
$$

is sufficiently small, where $i=2,3$. Thus for any $\mathcal{R} \in \bigcap_{\left(\lambda_{1}, \lambda_{2}, \lambda_{3}\right) \in \Lambda} C_{\lambda_{1}, \lambda_{2}, \lambda_{3}}$, we have $\mathcal{R} \in C_{\bar{\lambda}_{1}, \lambda_{2}, \lambda_{3}}$, where $\left(\bar{\lambda}_{1}, \lambda_{2}, \lambda_{3}\right) \in \Lambda$. It follows that

$$
\bar{\lambda}_{1} \mathcal{R}_{\alpha \alpha}+\left(1-\left(\bar{\lambda}_{1}+\lambda_{2}\right) \lambda_{2}\right) \mathcal{R}_{\beta \beta} \geq 0
$$

for arbitrary $1 \leq \alpha<\beta \leq N$ and $\left(\bar{\lambda}_{1}, \lambda_{2}, \lambda_{3}\right) \in \Lambda$, which implies

$$
\mathcal{R}_{11} \geq-\frac{1-\left(\bar{\lambda}_{1}+\lambda_{2}\right) \lambda_{2}}{\bar{\lambda}_{1}} \mathcal{R}_{22}
$$

for the above arbitrary $\left(\bar{\lambda}_{1}, \lambda_{2}, \lambda_{3}\right) \in \Lambda$. Then let $\lambda_{2}$ tend to

$$
\frac{\sqrt{\bar{\lambda}_{1}^{2}+4}-\bar{\lambda}_{1}}{2}
$$

decreasing monotonically such that $\left(\bar{\lambda}_{1}, \lambda_{2}, \lambda_{3}\right) \in \Lambda$. Then we have

$$
1-\left(\bar{\lambda}_{1}+\lambda_{2}\right) \lambda_{2} \rightarrow 0,
$$

which implies $\mathcal{R}_{11} \geq 0$. Since $\mathcal{R}_{11} \leq \mathcal{R}_{22} \leq \cdots \leq \mathcal{R}_{N N}$, it follows that

$$
\mathcal{R} \in\left\{\mathcal{R} \mid \mathcal{R}_{\alpha \alpha} \geq 0,1 \leq \alpha \leq N\right\} .
$$

Thus

$$
\bigcap_{\left(\lambda_{1}, \lambda_{2}, \lambda_{3}\right) \in \Lambda} C_{\lambda_{1}, \lambda_{2}, \lambda_{3}} \subset\left\{\mathcal{R} \mid \mathcal{R}_{\alpha \alpha} \geq 0,1 \leq \alpha \leq N\right\} .
$$


Secondly, we prove (5). For any $\left(\lambda_{1}, \lambda_{2}, \lambda_{3}\right) \in \Lambda$, if $\mathcal{R} \in C_{\lambda_{1}, \lambda_{2}, \lambda_{3}}$, then we have

$$
\lambda_{i} \mathcal{R}_{\alpha \alpha}+\left(1-\left(\lambda_{i}+\lambda_{j}\right) \lambda_{j}\right) \mathcal{R}_{\beta \beta} \geq 0
$$

for arbitrary $1 \leq \alpha<\beta \leq N$ and $1 \leq i \neq j \leq 3$. Then by the definition of $\Lambda$ and the choices of $\lambda_{1}, \lambda_{2}$, we have

$$
\begin{aligned}
\mathcal{R}_{\alpha \alpha}+\mathcal{R}_{\beta \beta} & =\frac{1}{\lambda_{1}}\left(\lambda_{1} \mathcal{R}_{\alpha \alpha}+\lambda_{1} \mathcal{R}_{\beta \beta}\right) \\
& \geq \lambda_{1} \mathcal{R}_{\alpha \alpha}+\left(1-\left(\lambda_{1}+\lambda_{2}\right) \lambda_{2}\right) \mathcal{R}_{\beta \beta} \\
& \geq 0
\end{aligned}
$$

Hence

$$
C_{\lambda_{1}, \lambda_{2}, \lambda_{3}} \subset\left\{\mathcal{R} \mid \mathcal{R}_{\alpha \alpha}+\mathcal{R}_{\beta \beta} \geq 0,1 \leq \alpha<\beta \leq N\right\}
$$

for any $\left(\lambda_{1}, \lambda_{2}, \lambda_{3}\right) \in \Lambda$, which implies

$$
\bigcup_{\left(\lambda_{1}, \lambda_{2}, \lambda_{3}\right) \in \Lambda} C_{\lambda_{1}, \lambda_{2}, \lambda_{3}} \subset\left\{\mathcal{R} \mid \mathcal{R}_{\alpha \alpha}+\mathcal{R}_{\beta \beta} \geq 0,1 \leq \alpha<\beta \leq N\right\} .
$$

Conversely, when $\lambda_{1}=1, \lambda_{2}=\lambda_{3}=0$, the 3 -parameter (1,0,0)-nonnegative curvature operator gives

$$
C_{1,0,0}=\left\{\mathcal{R} \mid \mathcal{R}_{\alpha \alpha}+\mathcal{R}_{\beta \beta} \geq 0,1 \leq \alpha<\beta \leq N\right\},
$$

which implies

$$
\bigcup_{\left(\lambda_{1}, \lambda_{2}, \lambda_{3}\right) \in \Lambda} C_{\lambda_{1}, \lambda_{2}, \lambda_{3}} \supset C_{1,0,0}=\left\{\mathcal{R} \mid \mathcal{R}_{\alpha \alpha}+\mathcal{R}_{\beta \beta} \geq 0,1 \leq \alpha<\beta \leq N\right\} .
$$

Now (7) and (8) imply (5).

The proof of (6) is similar to that of (5). We only need to note that

$$
\begin{aligned}
\mathcal{R}_{\alpha \alpha}+\mathcal{R}_{\beta \beta} & =\frac{1}{\lambda_{1}}\left(\lambda_{1} \mathcal{R}_{\alpha \alpha}+\lambda_{1} \mathcal{R}_{\beta \beta}\right) \\
& \geq \lambda_{1} \mathcal{R}_{\alpha \alpha}+\left(1-\left(\lambda_{1}+\lambda_{2}\right) \lambda_{2}\right) \mathcal{R}_{\beta \beta} \\
& >0,
\end{aligned}
$$

and when $\lambda_{1}=1, \lambda_{2}=\lambda_{3}=0$, the 3 -parameter $(1,0,0)$-positive curvature operator is $C_{1,0,0}=\left\{\mathcal{R} \mid \mathcal{R}_{\alpha \alpha}+\mathcal{R}_{\beta \beta}>0,1 \leq \alpha<\beta \leq N\right\}$.

Let $\left\{\varphi_{\alpha}\right\}_{\alpha=1}^{N}=\left\{e_{i} \wedge e_{j}\right\}_{i<j}$ be an orthonormal basis for $\wedge^{2} \mathbb{R}^{n}$, where each $\alpha$ corresponds to a pair $(i, j)$ with $i<j$. Then we have the following related rigidity property of manifolds with 3 -parameter $\lambda$-nonnegative curvature operators.

Theorem 2.2 (A rigidity property of scalar curvature for manifolds with 3-parameter $\lambda$-nonnegativity). The manifold with 3-parameter $\lambda$-nonnegativity has nonnegative scalar curvature $\operatorname{Scal}(\mathcal{R})$, and with equality if and only if $\mathcal{R}=0$. 
Proof. We compute

$$
\begin{aligned}
\operatorname{Tr}(\mathcal{R}) & =\sum_{\alpha=1}^{N}\left\langle\mathcal{R}\left(\varphi_{\alpha}\right), \varphi_{\alpha}\right\rangle \\
& =\sum_{i<j}\left\langle\mathcal{R}\left(e_{i} \wedge e_{j}\right), e_{i} \wedge e_{j}\right\rangle \\
& =\frac{1}{2} \sum_{i, j} \mathcal{R}_{i j i j} \\
& =\frac{1}{2} \operatorname{Tr}(\operatorname{Rc}(\mathcal{R})) \\
& =\frac{1}{2} \operatorname{Scal}(\mathcal{R}) .
\end{aligned}
$$

On the other hand, since $\mathcal{R}$ is 3 -parameter $\lambda$-nonnegative, we have

$$
\begin{aligned}
0 & \leq \sum_{1 \leq \alpha \neq \beta \neq \gamma \neq \delta \leq N} \mathcal{R}_{\alpha \alpha}+\lambda_{1} \mathcal{R}_{\beta \beta}+\lambda_{2} \mathcal{R}_{\gamma \gamma}+\lambda_{3} \mathcal{R}_{\delta \delta} \\
& =(N-1)(N-2)(N-3)\left(1+\lambda_{1}+\lambda_{2}+\lambda_{3}\right) \operatorname{Tr}(\mathcal{R}) \\
& =\frac{1}{2} \operatorname{Scal}(\mathcal{R})(N-1)(N-2)(N-3)\left(1+\lambda_{1}+\lambda_{2}+\lambda_{3}\right),
\end{aligned}
$$

which implies that $\operatorname{Scal}(\mathcal{R}) \geq 0$. Hence if $\operatorname{Scal}(\mathcal{R})=0$, then

$$
\mathcal{R}_{\alpha \alpha}+\lambda_{1} \mathcal{R}_{\beta \beta}+\lambda_{2} \mathcal{R}_{\gamma \gamma}+\lambda_{3} \mathcal{R}_{\delta \delta}=0
$$

for any $1 \leq \alpha \neq \beta \neq \gamma \neq \delta \leq N$, which implies that $\mathcal{R}_{\alpha \alpha}=0$ for any $1 \leq \alpha \leq N$.

Remark 6 . The fact in Theorem 2.2 was proven in [8] for nonnegative isotropic curvature. Recall that in [7], it is proved that 2-nonnegative curvature operator implies positive isotropic curvature. Thus Theorem 2.2 is in fact a direct corollary of a theorem of Micallef and Wang in [8].

\section{Proof of Theorem 1.4}

Note that the invariance under parallel translation of $C_{\lambda_{1}, \lambda_{2}, \lambda_{3}}$ is obvious.

For the convexity of $C_{\lambda_{1}, \lambda_{2}, \lambda_{3}}$, we use the fact that the sum of its first $k$ eigenvalues which is associated to the curvature operator matrix under the orthonormal basis of eigenvectors of $\mathcal{R}$ in $\mathfrak{s o}(n, \mathbb{R})$ is convex. Note that the inequalities defining $C_{\lambda_{1}, \lambda_{2}, \lambda_{3}}$ can be expressed as

$$
\begin{aligned}
& \mathcal{R}_{11}+\lambda_{1} \mathcal{R}_{22}+\lambda_{2} \mathcal{R}_{33}+\lambda_{3} \mathcal{R}_{44} \\
= & \lambda_{3}\left(\mathcal{R}_{11}+\mathcal{R}_{22}+\mathcal{R}_{33}+\mathcal{R}_{44}\right)+\left(\lambda_{2}-\lambda_{3}\right)\left(\mathcal{R}_{11}+\mathcal{R}_{22}+\mathcal{R}_{33}\right) \\
& +\left(\lambda_{1}-\lambda_{2}\right)\left(\mathcal{R}_{11}+\mathcal{R}_{22}\right)+\left(1-\lambda_{1}\right) \mathcal{R}_{11}
\end{aligned}
$$

and

$$
\lambda_{i} \mathcal{R}_{11}+\left(1-\left(\lambda_{i}+\lambda_{j}\right) \lambda_{j}\right) \mathcal{R}_{22}
$$




$$
=\left(1-\left(\lambda_{i}+\lambda_{j}\right) \lambda_{j}\right)\left(\mathcal{R}_{11}+\mathcal{R}_{22}\right)+\left(\lambda_{i}-1+\left(\lambda_{i}+\lambda_{j}\right) \lambda_{j}\right) \mathcal{R}_{11}
$$

which are two conical combinations of these convex functions. Because of the properties of $\Lambda$, we have that $\mathcal{R}_{11}+\lambda_{1} \mathcal{R}_{22}+\lambda_{2} \mathcal{R}_{33}+\lambda_{3} \mathcal{R}_{44}$ and $\lambda_{i} \mathcal{R}_{11}+$ $\left(1-\left(\lambda_{i}+\lambda_{j}\right) \lambda_{j}\right) \mathcal{R}_{22}$ are both convex.

Moreover, by the definition of $C_{\lambda_{1}, \lambda_{2}, \lambda_{3}}$, we actually have $0 \leq \mathcal{R}_{22} \leq \cdots \leq$ $\mathcal{R}_{N N}$, which implies any convex conical combination of $\mathcal{R}_{\alpha \alpha}, \mathcal{R}_{\beta \beta}, \mathcal{R}_{\gamma \gamma}, \mathcal{R}_{\delta \delta}$, where $2 \leq \alpha, \beta, \gamma, \delta \leq N$, is convex.

Then we turn to prove that $\mathcal{R}^{2}+\mathcal{R}^{\#}$ lies inside the tangent cone of the convex cone $C_{\lambda_{1}, \lambda_{2}, \lambda_{3}}$ of 3 -parameter $\lambda$-nonnegative curvature operators for any $\mathcal{R} \in \partial C_{\lambda_{1}, \lambda_{2}, \lambda_{3}}$.

Let $\left\{\omega_{\alpha}\right\}_{\alpha=1}^{N}$ be an orthonormal basis of eigenvectors of $\mathcal{R}$ in $\mathfrak{s o}(n, \mathbb{R})$ with corresponding eigenvalues $\mu_{1}(\mathcal{R}) \leq \mu_{2}(\mathcal{R}) \leq \cdots \leq \mu_{N}(\mathcal{R})$, where $N=\frac{n(n-1)}{2}$. Given $\mathcal{S} \in S_{B}^{2}(s o(n))$, let $\mathcal{S}_{\alpha \beta}=\mathcal{S}\left(\omega_{\alpha}, \omega_{\beta}\right)$. If $\mathcal{R} \in \partial C_{\lambda_{1}, \lambda_{2}, \lambda_{3}}$, then a vector $\mathcal{S}$ at the point $\mathcal{R}$ is in the tangent cone of $C_{\lambda_{1}, \lambda_{2}, \lambda_{3}}$ if and only if

(i) $\mathcal{S}_{\alpha \alpha}+\lambda_{1} \mathcal{S}_{\beta \beta}+\lambda_{2} \mathcal{S}_{\gamma \gamma}+\lambda_{3} \mathcal{S}_{\delta \delta} \geq 0$ for arbitrary $1 \leq \alpha<\beta<\gamma<\delta \leq N$ such that

and

$$
\mathcal{R}_{\alpha \alpha}+\lambda_{1} \mathcal{R}_{\beta \beta}+\lambda_{2} \mathcal{R}_{\gamma \gamma}+\lambda_{3} \mathcal{R}_{\delta \delta}=0
$$

where $1 \leq i \neq j \leq 3$

$$
\lambda_{i} \mathcal{R}_{\alpha \alpha}+\left(1-\left(\lambda_{i}+\lambda_{j}\right) \lambda_{j}\right) \mathcal{R}_{\beta \beta}=0,
$$

(ii) $\lambda_{i} \mathcal{S}_{\alpha \alpha}+\left(1-\left(\lambda_{i}+\lambda_{j}\right) \lambda_{j}\right) \mathcal{S}_{\beta \beta} \geq 0$ for arbitrary $1 \leq \alpha<\beta \leq N$ such that

and

$$
\mathcal{R}_{\alpha \alpha}+\lambda_{1} \mathcal{R}_{\beta \beta}+\lambda_{2} \mathcal{R}_{\gamma \gamma}+\lambda_{3} \mathcal{R}_{\delta \delta}=0
$$

where $1 \leq i \neq j \leq 3$.

$$
\lambda_{i} \mathcal{R}_{\alpha \alpha}+\left(1-\left(\lambda_{i}+\lambda_{j}\right) \lambda_{j}\right) \mathcal{R}_{\beta \beta}=0,
$$

Since $\left\{\omega_{\alpha}\right\}_{\alpha=1}^{N}$ is an orthonormal basis of eigenvectors of $\mathcal{R}$ in $\mathfrak{s o}(n, \mathbb{R})$ with corresponding eigenvalues $\mu_{1}(\mathcal{R}) \leq \mu_{2}(\mathcal{R}) \leq \cdots \leq \mu_{N}(\mathcal{R})$, we have $\mathcal{R}_{11} \leq$ $\mathcal{R}_{22} \leq \cdots \leq \mathcal{R}_{N N}$, where $\mu_{\alpha}(\mathcal{R})=\mathcal{R}\left(\omega_{\alpha}, \omega_{\alpha}\right)=\mathcal{R}_{\alpha \alpha}$. Thus

$$
\mathcal{R}_{\alpha \alpha}+\lambda_{1} \mathcal{R}_{\beta \beta}+\lambda_{2} \mathcal{R}_{\gamma \gamma}+\lambda_{3} \mathcal{R}_{\delta \delta} \geq \mathcal{R}_{11}+\lambda_{1} \mathcal{R}_{22}+\lambda_{2} \mathcal{R}_{33}+\lambda_{3} \mathcal{R}_{44}
$$

and

$$
\lambda_{i} \mathcal{R}_{\alpha \alpha}+\left(1-\left(\lambda_{i}+\lambda_{j}\right) \lambda_{j}\right) \mathcal{R}_{\beta \beta} \geq \lambda_{i} \mathcal{R}_{11}+\left(1-\left(\lambda_{i}+\lambda_{j}\right) \lambda_{j}\right) \mathcal{R}_{22}
$$

for arbitrary $1 \leq \alpha<\beta<\gamma<\delta \leq N$ and $1 \leq i \neq j \leq 3$.

Hence we only need to prove

$$
\mathcal{R}_{11}+\lambda_{1} \mathcal{R}_{22}+\lambda_{2} \mathcal{R}_{33}+\lambda_{3} \mathcal{R}_{44} \geq 0
$$

and

$$
\lambda_{i} \mathcal{R}_{11}+\left(1-\left(\lambda_{i}+\lambda_{j}\right) \lambda_{j}\right) \mathcal{R}_{22} \geq 0
$$

where $1 \leq i \neq j \leq 3$, are preserved by the ODE $d \mathcal{R} / d t=\mathcal{R}^{2}+\mathcal{R}^{\#}$. Note that a convex set is preserved by the flow of a vector field if and only if at each point of the boundary of the convex set, the vector field points towards the inside 
of the convex set. Then the proof of Theorem 1.4 is reduced to the following Claim 3.1 (see also [3]):

Claim 3.1. (i) If

$$
\mathcal{R}_{\alpha \alpha}+\lambda_{1} \mathcal{R}_{\beta \beta}+\lambda_{2} \mathcal{R}_{\gamma \gamma}+\lambda_{3} \mathcal{R}_{\delta \delta}=0
$$

and

$$
\lambda_{i} \mathcal{R}_{\alpha \alpha}+\left(1-\left(\lambda_{i}+\lambda_{j}\right) \lambda_{j}\right) \mathcal{R}_{\beta \beta} \geq 0
$$

for arbitrary $1 \leq \alpha<\beta<\gamma<\delta \leq N,\left(\lambda_{1}, \lambda_{2}, \lambda_{3}\right) \in \Lambda$ and $1 \leq i \neq j \leq 3$, then $\left(\mathcal{R}^{2}+\mathcal{R}^{\#}\right)_{\alpha \alpha}+\lambda_{1}\left(\mathcal{R}^{2}+\mathcal{R}^{\#}\right)_{\beta \beta}+\lambda_{2}\left(\mathcal{R}^{2}+\mathcal{R}^{\#}\right)_{\gamma \gamma}+\lambda_{3}\left(\mathcal{R}^{2}+\mathcal{R}^{\#}\right)_{\delta \delta} \geq 0$, where $\left(\mathcal{R}^{2}+\mathcal{R}^{\#}\right)_{\alpha \alpha}=\left(\mathcal{R}^{2}+\mathcal{R}^{\#}\right)\left(\omega_{\alpha}, \omega_{\alpha}\right)$.

(ii) If

and

$$
\mathcal{R}_{\alpha \alpha}+\lambda_{1} \mathcal{R}_{\beta \beta}+\lambda_{2} \mathcal{R}_{\gamma \gamma}+\lambda_{3} \mathcal{R}_{\delta \delta} \geq 0
$$

$$
\lambda_{i} \mathcal{R}_{\alpha \alpha}+\left(1-\left(\lambda_{i}+\lambda_{j}\right) \lambda_{j}\right) \mathcal{R}_{\beta \beta}=0
$$

for arbitrary $1 \leq \alpha<\beta<\gamma<\delta \leq N,\left(\lambda_{1}, \lambda_{2}, \lambda_{3}\right) \in \Lambda$ and $1 \leq i \neq j \leq 3$, then

$$
\lambda_{i}\left(\mathcal{R}^{2}+\mathcal{R}^{\#}\right)_{\alpha \alpha}+\left(1-\left(\lambda_{i}+\lambda_{j}\right) \lambda_{j}\right)\left(\mathcal{R}^{2}+\mathcal{R}^{\#}\right)_{\beta \beta} \geq 0 .
$$

Proof. (i) By calculation, we see

$$
\begin{aligned}
& \left(\mathcal{R}^{2}+\mathcal{R}^{\#}\right)_{11}+\lambda_{1}\left(\mathcal{R}^{2}+\mathcal{R}^{\#}\right)_{22}+\lambda_{2}\left(\mathcal{R}^{2}+\mathcal{R}^{\#}\right)_{33}+\lambda_{3}\left(\mathcal{R}^{2}+\mathcal{R}^{\#}\right)_{44} \\
= & \mu_{1}^{2}+\lambda_{1} \mu_{2}^{2}+\lambda_{2} \mu_{3}^{2}+\lambda_{3} \mu_{4}^{2} \\
& +2 \sum_{\alpha<\beta}\left(\left(c_{1}^{\alpha \beta}\right)^{2}+\lambda_{1}\left(c_{2}^{\alpha \beta}\right)^{2}+\lambda_{2}\left(c_{3}^{\alpha \beta}\right)^{2}+\lambda_{3}\left(c_{4}^{\alpha \beta}\right)^{2}\right) \mu_{\alpha} \mu_{\beta} .
\end{aligned}
$$

We only need to prove the right-hand side of the equality is nonnegative. In fact,

$$
\begin{aligned}
& \sum_{\alpha<\beta}\left(\left(c_{1}^{\alpha \beta}\right)^{2}+\lambda_{1}\left(c_{2}^{\alpha \beta}\right)^{2}+\lambda_{2}\left(c_{3}^{\alpha \beta}\right)^{2}+\lambda_{3}\left(c_{4}^{\alpha \beta}\right)^{2}\right) \mu_{\alpha} \mu_{\beta} \\
= & \sum_{2 \leq \alpha<\beta}\left(c_{1}^{\alpha \beta}\right)^{2} \mu_{\alpha} \mu_{\beta}+\sum_{1 \leq \alpha<\beta} \lambda_{1}\left(c_{2}^{\alpha \beta}\right)^{2} \mu_{\alpha} \mu_{\beta}+\sum_{1 \leq \alpha<\beta} \lambda_{2}\left(c_{3}^{\alpha \beta}\right)^{2} \mu_{\alpha} \mu_{\beta} \\
& +\sum_{1 \leq \alpha<\beta} \lambda_{3}\left(c_{4}^{\alpha \beta}\right)^{2} \mu_{\alpha} \mu_{\beta} \\
= & \sum_{\beta>2}\left(c_{1}^{2 \beta}\right)^{2} \mu_{2} \mu_{\beta}+\sum_{\beta>3}\left(c_{1}^{3 \beta}\right)^{2} \mu_{3} \mu_{\beta}+\sum_{\beta>4}\left(c_{1}^{4 \beta}\right)^{2} \mu_{4} \mu_{\beta} \\
& +\sum_{5 \leq \alpha<\beta}\left(c_{1}^{\alpha \beta}\right)^{2} \mu_{\alpha} \mu_{\beta}+\lambda_{1} \sum_{\beta>2}\left(c_{2}^{1 \beta}\right)^{2} \mu_{1} \mu_{\beta}+\lambda_{1} \sum_{\beta>3}\left(c_{2}^{3 \beta}\right)^{2} \mu_{3} \mu_{\beta} \\
& +\lambda_{1} \sum_{\beta>4}\left(c_{2}^{4 \beta}\right)^{2} \mu_{4} \mu_{\beta}+\lambda_{1} \sum_{5 \leq \alpha<\beta}\left(c_{2}^{\alpha \beta}\right)^{2} \mu_{\alpha} \mu_{\beta}+\lambda_{2}\left(c_{3}^{12}\right)^{2} \mu_{1} \mu_{2}
\end{aligned}
$$


A NEW 3-PARAMETER CURVATURE CONDITION PRESERVED BY RICCI FLOW 839

$$
\begin{aligned}
& +\lambda_{2} \sum_{\beta>3}\left(c_{3}^{1 \beta}\right)^{2} \mu_{1} \mu_{\beta}+\lambda_{2} \sum_{\beta>3}\left(c_{3}^{2 \beta}\right)^{2} \mu_{2} \mu_{\beta}+\lambda_{2} \sum_{\beta>4}\left(c_{3}^{4 \beta}\right)^{2} \mu_{4} \mu_{\beta} \\
& +\lambda_{2} \sum_{5 \leq \alpha<\beta}\left(c_{3}^{\alpha \beta}\right)^{2} \mu_{\alpha} \mu_{\beta}+\lambda_{3}\left(c_{4}^{12}\right)^{2} \mu_{1} \mu_{2}+\lambda_{3}\left(c_{4}^{13}\right)^{2} \mu_{1} \mu_{3} \\
& +\lambda_{3} \sum_{\beta>4}\left(c_{4}^{1 \beta}\right)^{2} \mu_{1} \mu_{\beta}+\lambda_{3}\left(c_{4}^{23}\right)^{2} \mu_{2} \mu_{3}+\lambda_{3} \sum_{\beta>4}\left(c_{4}^{2 \beta}\right)^{2} \mu_{2} \mu_{\beta} \\
& +\lambda_{3} \sum_{\beta>4}\left(c_{4}^{3 \beta}\right)^{2} \mu_{3} \mu_{\beta}+\lambda_{3} \sum_{5 \leq \alpha<\beta}\left(c_{4}^{\alpha \beta}\right)^{2} \mu_{\alpha} \mu_{\beta} \\
= & \left(c_{1}^{23}\right)^{2}\left(\mu_{2} \mu_{3}+\lambda_{1} \mu_{1} \mu_{3}+\lambda_{2} \mu_{1} \mu_{2}\right)+\left(c_{1}^{24}\right)^{2}\left(\mu_{2} \mu_{4}+\lambda_{1} \mu_{1} \mu_{4}+\lambda_{3} \mu_{1} \mu_{2}\right) \\
& +\left(c_{1}^{34}\right)^{2}\left(\mu_{3} \mu_{4}+\lambda_{2} \mu_{1} \mu_{4}+\lambda_{3} \mu_{1} \mu_{3}\right)+\left(c_{2}^{34}\right)^{2}\left(\lambda_{1} \mu_{3} \mu_{4}+\lambda_{2} \mu_{2} \mu_{4}+\lambda_{3} \mu_{2} \mu_{3}\right) \\
& +\sum_{\beta>4}\left(c_{1}^{2 \beta}\right)^{2}\left(\mu_{2}+\lambda_{1} \mu_{1}\right) \mu_{\beta}+\sum_{\beta>4}\left(c_{1}^{3 \beta}\right)^{2}\left(\mu_{3}+\lambda_{2} \mu_{1}\right) \mu_{\beta} \\
& +\sum_{\beta>4}\left(c_{1}^{4 \beta}\right)^{2}\left(\mu_{4}+\lambda_{3} \mu_{1}\right) \mu_{\beta}+\sum_{\beta>4}\left(c_{2}^{3 \beta}\right)^{2}\left(\lambda_{1} \mu_{3}+\lambda_{2} \mu_{2}\right) \mu_{\beta} \\
& +\sum_{\beta>4}\left(c_{2}^{4 \beta}\right)^{2}\left(\lambda_{1} \mu_{4}+\lambda_{3} \mu_{2}\right) \mu_{\beta}+\sum_{\beta>4}\left(c_{3}^{4 \beta}\right)^{2}\left(\lambda_{2} \mu_{4}+\lambda_{3} \mu_{3}\right) \mu_{\beta} \\
& +\sum_{5 \leq \alpha<\beta}\left(c_{1}^{\alpha \beta}\right)^{2} \mu_{\alpha} \mu_{\beta}+\lambda_{1} \sum_{5 \leq \alpha<\beta}\left(c_{2}^{\alpha \beta}\right)^{2} \mu_{\alpha} \mu_{\beta} \\
& +\lambda_{2} \sum_{5 \leq \alpha<\beta}\left(c_{3}^{\alpha \beta}\right)^{2} \mu_{\alpha} \mu_{\beta}+\lambda_{3} \sum_{5 \leq \alpha<\beta}\left(c_{4}^{\alpha \beta}\right)^{2} \mu_{\alpha} \mu_{\beta} .
\end{aligned}
$$

Since $\mu_{2}+\lambda_{1} \mu_{1} \geq\left(1-\left(\lambda_{1}+\lambda_{2}\right) \lambda_{2}\right) \mu_{2}+\lambda_{1} \mu_{1} \geq 0$, we have

$$
\sum_{\beta>4}\left(c_{1}^{2 \beta}\right)^{2}\left(\mu_{2}+\lambda_{1} \mu_{1}\right) \mu_{\beta} \geq 0
$$

For the same reason, we also have

$$
\sum_{\beta>4}\left(c_{1}^{3 \beta}\right)^{2}\left(\mu_{3}+\lambda_{2} \mu_{1}\right) \mu_{\beta} \geq 0
$$

and

$$
\sum_{\beta>4}\left(c_{1}^{4 \beta}\right)^{2}\left(\mu_{4}+\lambda_{3} \mu_{1}\right) \mu_{\beta} \geq 0
$$

Since

$$
\begin{aligned}
& \mu_{2} \mu_{3}+\lambda_{1} \mu_{1} \mu_{3}+\lambda_{2} \mu_{1} \mu_{2} \\
= & \lambda_{2} \mu_{2}\left(\mu_{1}+\left(\lambda_{1}+\lambda_{2}\right) \mu_{3}\right)+\mu_{3}\left(\left(1-\left(\lambda_{1}+\lambda_{2}\right) \lambda_{2}\right) \mu_{2}+\lambda_{1} \mu_{1}\right) \\
\geq & 0
\end{aligned}
$$


it follows that $\left(c_{1}^{23}\right)^{2}\left(\mu_{2} \mu_{3}+\lambda_{1} \mu_{1} \mu_{3}+\lambda_{2} \mu_{1} \mu_{2}\right) \geq 0$.

Then as in the proof above, we also have

$$
\left(c_{1}^{24}\right)^{2}\left(\mu_{2} \mu_{4}+\lambda_{1} \mu_{1} \mu_{4}+\lambda_{3} \mu_{1} \mu_{2}\right) \geq 0
$$

and

$$
\left(c_{1}^{34}\right)^{2}\left(\mu_{3} \mu_{4}+\lambda_{2} \mu_{1} \mu_{4}+\lambda_{3} \mu_{1} \mu_{3}\right) \geq 0 .
$$

Moreover, by the fact that $\mu_{1} \leq 0 \leq \mu_{2} \leq \cdots \leq \mu_{N}$, we can also get

$$
\begin{aligned}
& \left(c_{2}^{34}\right)^{2}\left(\lambda_{1} \mu_{3} \mu_{4}+\lambda_{2} \mu_{2} \mu_{4}+\lambda_{3} \mu_{2} \mu_{3}\right)+\sum_{\beta>4}\left(c_{2}^{3 \beta}\right)^{2}\left(\lambda_{1} \mu_{3}+\lambda_{2} \mu_{2}\right) \mu_{\beta} \\
& +\sum_{\beta>4}\left(c_{2}^{4 \beta}\right)^{2}\left(\lambda_{1} \mu_{4}+\lambda_{3} \mu_{2}\right) \mu_{\beta}+\sum_{\beta>4}\left(c_{3}^{4 \beta}\right)^{2}\left(\lambda_{2} \mu_{4}+\lambda_{3} \mu_{3}\right) \mu_{\beta} \\
& +\sum_{5 \leq \alpha<\beta}\left(c_{1}^{\alpha \beta}\right)^{2} \mu_{\alpha} \mu_{\beta}+\lambda_{1} \sum_{5 \leq \alpha<\beta}\left(c_{2}^{\alpha \beta}\right)^{2} \mu_{\alpha} \mu_{\beta}+\lambda_{2} \sum_{5 \leq \alpha<\beta}\left(c_{3}^{\alpha \beta}\right)^{2} \mu_{\alpha} \mu_{\beta} \\
& +\lambda_{3} \sum_{5 \leq \alpha<\beta}\left(c_{4}^{\alpha \beta}\right)^{2} \mu_{\alpha} \mu_{\beta} \geq 0 .
\end{aligned}
$$

Thus all of above lead to

$$
\sum_{\alpha<\beta}\left(\left(c_{1}^{\alpha \beta}\right)^{2}+\lambda_{1}\left(c_{2}^{\alpha \beta}\right)^{2}+\lambda_{2}\left(c_{3}^{\alpha \beta}\right)^{2}+\lambda_{3}\left(c_{4}^{\alpha \beta}\right)^{2}\right) \mu_{\alpha} \mu_{\beta} \geq 0,
$$

which is to say that

$$
\left(\mathcal{R}^{2}+\mathcal{R}^{\#}\right)_{11}+\lambda_{1}\left(\mathcal{R}^{2}+\mathcal{R}^{\#}\right)_{22}+\lambda_{2}\left(\mathcal{R}^{2}+\mathcal{R}^{\#}\right)_{33}+\lambda_{3}\left(\mathcal{R}^{2}+\mathcal{R}^{\#}\right)_{44} \geq 0
$$

(ii) As in the proof of (i), without loss of generality, we only need to consider $\left(\lambda_{i}, \lambda_{j}\right)=\left(\lambda_{1}, \lambda_{2}\right)$. Let $\gamma=\lambda_{1}$ and $\delta=1-\left(\lambda_{1}+\lambda_{2}\right) \lambda_{2}$. By a direct calculation, we have

$$
\begin{aligned}
& \lambda_{1}\left(\mathcal{R}^{2}+\mathcal{R}^{\#}\right)_{11}+\left(1-\left(\lambda_{1}+\lambda_{2}\right) \lambda_{2}\right)\left(\mathcal{R}^{2}+\mathcal{R}^{\#}\right)_{22} \\
= & \gamma \mu_{1}^{2}+\delta \mu_{2}^{2}+2 \sum_{\alpha<\beta}\left(\gamma\left(c_{1}^{\alpha \beta}\right)^{2}+\delta\left(c_{2}^{\alpha \beta}\right)^{2}\right) \mu_{\alpha} \mu_{\beta} .
\end{aligned}
$$

Then we only need to prove the right-hand side is nonnegative. In fact,

$$
\begin{aligned}
& \sum_{\alpha<\beta}\left(\gamma\left(c_{1}^{\alpha \beta}\right)^{2}+\delta\left(c_{2}^{\alpha \beta}\right)^{2}\right) \mu_{\alpha} \mu_{\beta} \\
= & \gamma \sum_{2 \leq \alpha<\beta}\left(c_{1}^{\alpha \beta}\right)^{2} \mu_{\alpha} \mu_{\beta}+\delta \sum_{1 \leq \alpha<\beta}\left(c_{2}^{\alpha \beta}\right)^{2} \mu_{\alpha} \mu_{\beta} \\
= & \gamma \sum_{\beta>2}\left(c_{1}^{2 \beta}\right)^{2} \mu_{2} \mu_{\beta}+\delta \sum_{\beta>2}\left(c_{2}^{1 \beta}\right)^{2} \mu_{1} \mu_{\beta}+\gamma \sum_{3 \leq \alpha<\beta}\left(c_{1}^{\alpha \beta}\right)^{2} \mu_{\alpha} \mu_{\beta} \\
& +\delta \sum_{3 \leq \alpha<\beta}\left(c_{2}^{\alpha \beta}\right)^{2} \mu_{\alpha} \mu_{\beta}
\end{aligned}
$$


A NEW 3-PARAMETER CURVATURE CONDITION PRESERVED BY RICCI FLOW 841

$$
\begin{aligned}
& =\sum_{\beta>2}\left(c_{1}^{2 \beta}\right)^{2}\left(\gamma \mu_{2}+\delta \mu_{1}\right) \mu_{\beta}+\gamma \sum_{3 \leq \alpha<\beta}\left(c_{1}^{\alpha \beta}\right)^{2} \mu_{\alpha} \mu_{\beta}+\delta \sum_{3 \leq \alpha<\beta}\left(c_{2}^{\alpha \beta}\right)^{2} \mu_{\alpha} \mu_{\beta} \\
& =\delta \sum_{\beta>2}\left(c_{1}^{2 \beta}\right)^{2}\left(\frac{\gamma}{\delta} \mu_{2}+\mu_{1}\right) \mu_{\beta}+\gamma \sum_{3 \leq \alpha<\beta}\left(c_{1}^{\alpha \beta}\right)^{2} \mu_{\alpha} \mu_{\beta}+\delta \sum_{3 \leq \alpha<\beta}\left(c_{2}^{\alpha \beta}\right)^{2} \mu_{\alpha} \mu_{\beta} .
\end{aligned}
$$

Since $\gamma=\lambda_{1} \geq 1-\left(\lambda_{1}+\lambda_{2}\right) \lambda_{2}=\delta>0$, we have

$$
\begin{aligned}
& \sum_{\alpha<\beta}\left(\gamma\left(c_{1}^{\alpha \beta}\right)^{2}+\delta\left(c_{2}^{\alpha \beta}\right)^{2}\right) \mu_{\alpha} \mu_{\beta} \\
\geq & \delta \sum_{\beta>2}\left(c_{1}^{2 \beta}\right)^{2}\left(\frac{\delta}{\gamma} \mu_{2}+\mu_{1}\right) \mu_{\beta}+\gamma \sum_{3 \leq \alpha<\beta}\left(c_{1}^{\alpha \beta}\right)^{2} \mu_{\alpha} \mu_{\beta}+\delta \sum_{3 \leq \alpha<\beta}\left(c_{2}^{\alpha \beta}\right)^{2} \mu_{\alpha} \mu_{\beta} \\
\geq & 0 .
\end{aligned}
$$

\section{The strong maximum principle for 3-parameter $\lambda$-nonnegativity}

Theorem 4.1 (Strong maximum principle for 3-parameter $\lambda$-nonnegativity). Let $\left(M^{n}, g(t)\right), t \in[0, T)$ be a solution to the Ricci flow on a closed manifold. If the curvature operator $\mathcal{R}(g(0))$ is 3-parameter $\lambda$-nonnegative, then

(i) For any $t>0$, the curvature operator $\mathcal{R}(g(t))$ is either nonnegative or 3-parameter $\lambda$-positive.

(ii) If $\mathcal{R}(g(0))$ is 3-parameter $\lambda$-positive at a point in $M^{n}$, then $\mathcal{R}(g(t))$ is 3-parameter $\lambda$-positive everywhere for $0<t<T$.

Proof. By Theorem 1.4, we have $\mathcal{R}(g(t))$ is also 3-parameter $\lambda$-nonnegative for all $t>0$. We will prove (ii) first.

(ii) As in the proof of Theorem 1.4 we only need to consider $(\alpha, \beta, \gamma, \delta)=$ $(1,2,3,4)$. Let $\varphi(x)$ be a smooth nonnegative function such that

$$
\varphi(x) \leq \frac{\mu_{1}(\mathcal{R}(x, 0))+\lambda_{1} \mu_{2}(\mathcal{R}(x, 0))+\lambda_{2} \mu_{3}(\mathcal{R}(x, 0))+\lambda_{3} \mu_{4}(\mathcal{R}(x, 0))}{1+\lambda_{1}+\lambda_{2}+\lambda_{3}}
$$

and

$$
\varphi(x) \leq \frac{\lambda_{i} \mu_{1}(\mathcal{R}(x, 0))+\left(1-\left(\lambda_{i}+\lambda_{j}\right) \lambda_{j}\right) \mu_{2}(\mathcal{R}(x, 0))}{\lambda_{i}+\left(1-\left(\lambda_{i}+\lambda_{j}\right) \lambda_{j}\right)}
$$

for all $x \in M^{n}$ and $1 \leq i \neq j \leq 3$. We also assume that there exists $x_{0} \in M^{n}$ such that

$$
\varphi\left(x_{0}\right) \geq \frac{\mu_{1}\left(\mathcal{R}\left(x_{0}, 0\right)\right)+\lambda_{1} \mu_{2}\left(\mathcal{R}\left(x_{0}, 0\right)\right)+\lambda_{2} \mu_{3}\left(\mathcal{R}\left(x_{0}, 0\right)\right)+\lambda_{3} \mu_{4}(\mathcal{R}(x, 0))}{2\left(1+\lambda_{1}+\lambda_{2}+\lambda_{3}\right)}
$$

and

$$
\varphi\left(x_{0}\right) \geq \frac{\lambda_{i} \mu_{1}\left(\mathcal{R}\left(x_{0}, 0\right)\right)+\left(1-\left(\lambda_{i}+\lambda_{j}\right) \lambda_{j}\right) \mu_{2}\left(\mathcal{R}\left(x_{0}, 0\right)\right)}{2\left(\lambda_{i}+\left(1-\left(\lambda_{i}+\lambda_{j}\right) \lambda_{j}\right)\right)} .
$$

Let $f(x, t)$ be a solution to

$$
\frac{\partial f}{\partial t}=\Delta f-A f
$$


such that $f(x, 0)=\varphi(x)$. Define

$$
\tilde{\mathcal{R}}(x, t)=\mathcal{R}(x, t)+\left(\varepsilon e^{A t}-f(x, t)\right) i d_{\wedge^{2}}(x),
$$

where $\varepsilon>0$. For $A$ sufficiently large, with Ricci flow equation

$$
\frac{\partial}{\partial t} g=-2 \operatorname{Ric}(g)
$$

we can prove that (see [3])

$$
\frac{\partial}{\partial t} \tilde{\mathcal{R}}>\Delta \tilde{\mathcal{R}}+\tilde{\mathcal{R}}^{2}+\tilde{\mathcal{R}}^{\#}
$$

for $\varepsilon \in\left(0, e^{-A T}\right]$. Moreover, when $t=0$, we have

$$
\tilde{\mathcal{R}}(x, 0)=\mathcal{R}(x, 0)+(\varepsilon-f(x, 0)) i d_{\wedge^{2}}(x)=\mathcal{R}(x, 0)+(\varepsilon-\varphi(x)) i d_{\wedge^{2}}(x) .
$$

By using the definition of $\varphi(x)$, we have

$$
\begin{aligned}
& (\tilde{\mathcal{R}}(x, 0))_{11}+\lambda_{1}(\tilde{\mathcal{R}}(x, 0))_{22}+\lambda_{2}(\tilde{\mathcal{R}}(x, 0))_{33}+\lambda_{3}(\tilde{\mathcal{R}}(x, 0))_{44} \\
= & \mu_{1}(\mathcal{R}(x, 0))+\lambda_{1} \mu_{2}(\mathcal{R}(x, 0))+\lambda_{2} \mu_{3}(\mathcal{R}(x, 0))+\lambda_{3} \mu_{4}(\mathcal{R}(x, 0)) \\
& -\left(1+\lambda_{1}+\lambda_{2}+\lambda_{3}\right) \varphi(x)+\left(1+\lambda_{1}+\lambda_{2}+\lambda_{3}\right) \varepsilon \\
> & 0
\end{aligned}
$$

and

$$
\begin{aligned}
& \lambda_{i}(\tilde{\mathcal{R}}(x, 0))_{11}+\left(1-\left(\lambda_{i}+\lambda_{j}\right) \lambda_{j}\right)(\tilde{\mathcal{R}}(x, 0))_{22} \\
= & \lambda_{i} \mu_{1}(\mathcal{R}(x, 0))+\left(1-\left(\lambda_{i}+\lambda_{j}\right) \lambda_{j}\right) \mu_{2}(\mathcal{R}(x, 0)) \\
& -\left(\lambda_{i}+\left(1-\left(\lambda_{i}+\lambda_{j}\right) \lambda_{j}\right)\right) \varphi(x)+\left(\lambda_{i}+\left(1-\left(\lambda_{i}+\lambda_{j}\right) \lambda_{j}\right)\right) \varepsilon \\
> & 0
\end{aligned}
$$

for any $1 \leq i \neq j \leq 3$.

Then applying Theorem 1.5 and 1.6 to (9), we have

$$
(\tilde{\mathcal{R}}(x, t))_{11}+\lambda_{1}(\tilde{\mathcal{R}}(x, t))_{22}+\lambda_{2}(\tilde{\mathcal{R}}(x, t))_{33}+\lambda_{3}(\tilde{\mathcal{R}}(x, t))_{44} \geq 0
$$

and

$$
\lambda_{i}(\tilde{\mathcal{R}}(x, t))_{11}+\left(1-\left(\lambda_{i}+\lambda_{j}\right) \lambda_{j}\right)(\tilde{\mathcal{R}}(x, t))_{22} \geq 0
$$

for all $t>0$. Thus taking the limit as $\varepsilon \rightarrow 0$, we conclude that

$$
\begin{aligned}
& \mu_{1}(\mathcal{R}(x, t))+\lambda_{1} \mu_{2}(\mathcal{R}(x, t))+\lambda_{2} \mu_{3}(\mathcal{R}(x, t))+\lambda_{3} \mu_{4}(\mathcal{R}(x, t)) \\
& -\left(1+\lambda_{1}+\lambda_{2}+\lambda_{3}\right) f(x, t) i d_{\wedge^{2}}(x) \geq 0
\end{aligned}
$$

and

$$
\begin{aligned}
& \lambda_{i} \mu_{1}(\mathcal{R}(x, t))+\left(1-\left(\lambda_{i}+\lambda_{j}\right) \lambda_{j}\right) \mu_{2}(\mathcal{R}(x, t)) \\
& -\left(\lambda_{i}+\left(1-\left(\lambda_{i}+\lambda_{j}\right) \lambda_{j}\right)\right) f(x, t) i d_{\wedge^{2}}(x) \geq 0
\end{aligned}
$$

for arbitrary $(x, t) \in M^{n} \times[0, T)$. 
On the other hand, since $f(x, t)$ is a solution to the parabolic equation

$$
\frac{\partial f}{\partial t}=\Delta f-A f
$$

such that $f\left(x_{0}, 0\right)=\varphi\left(x_{0}\right)>0$, by the strong maximum principle for the parabolic equation, we have $f(x, t)>0$ for arbitrary $(x, t) \in M^{n} \times[0, T)$. Hence

$$
\mu_{1}(\mathcal{R}(x, t))+\lambda_{1} \mu_{2}(\mathcal{R}(x, t))+\lambda_{2} \mu_{3}(\mathcal{R}(x, t))+\lambda_{3} \mu_{4}(\mathcal{R}(x, t))>0
$$

and

$$
\lambda_{i} \mu_{1}(\mathcal{R}(x, t))+\left(1-\left(\lambda_{i}+\lambda_{j}\right) \lambda_{j}\right) \mu_{2}(\mathcal{R}(x, t))>0
$$

for arbitrary $(x, t) \in M^{n} \times[0, T)$ and $1 \leq i \neq j \leq 3$.

(i) By (ii), if $g\left(t_{1}\right)$ is 3-parameter $\lambda$-nonnegative everywhere in $M^{n}$ and 3parameter $\lambda$-positive at a point in $M^{n}$, then $g(t)$ is 3 -parameter $\lambda$-positive everywhere for $t>t_{1}$.

As in the proof of (ii), without loss of generality, we only need to consider $(\alpha, \beta, \gamma, \delta)=(1,2,3,4)$. Hence if for some $t_{0}>0$, we have

$$
\mu_{1}\left(\mathcal{R}\left(x_{0}, t_{0}\right)\right)+\lambda_{1} \mu_{2}\left(\mathcal{R}\left(x_{0}, t_{0}\right)\right)+\lambda_{2} \mu_{3}\left(\mathcal{R}\left(x_{0}, t_{0}\right)\right)+\lambda_{3} \mu_{4}\left(\mathcal{R}\left(x_{0}, t_{0}\right)\right)=0
$$

or

$$
\lambda_{i} \mu_{1}\left(\mathcal{R}\left(x_{0}, t_{0}\right)\right)+\left(1-\left(\lambda_{i}+\lambda_{j}\right) \lambda_{j}\right) \mu_{2}\left(\mathcal{R}\left(x_{0}, t_{0}\right)\right)=0
$$

at point $x_{0}$ and for $1 \leq i \neq j \leq 3$, then we consider the following two cases:

If (10) is satisfied, by (ii), we have

$$
\mu_{1}(\mathcal{R}(x, t))+\lambda_{1} \mu_{2}(\mathcal{R}(x, t))+\lambda_{2} \mu_{3}(\mathcal{R}(x, t))+\lambda_{3} \mu_{4}(\mathcal{R}(x, t))=0
$$

for arbitrary $(x, t) \in M^{n} \times\left[0, t_{0}\right]$. We will prove the following result:

$$
\mu_{1}(\mathcal{R}(x, t))=\mu_{2}(\mathcal{R}(x, t))=\mu_{3}(\mathcal{R}(x, t))=\mu_{4}(\mathcal{R}(x, t))=0
$$

for arbitrary $(x, t) \in M^{n} \times\left[0, t_{0}\right]$.

To prove (12), pick some $\left(x_{1}, t_{1}\right)$ and let $\omega_{1}, \omega_{2}, \omega_{3}$ and $\omega_{4}$ be the unit 2forms at $\left(x_{1}, t_{1}\right)$, which are the eigenvectors for $\mathcal{R}\left(x_{1}, t_{1}\right)$ corresponding to $\mu_{1}\left(\mathcal{R}\left(x_{1}, t_{1}\right)\right), \mu_{2}\left(\mathcal{R}\left(x_{1}, t_{1}\right)\right), \mu_{3}\left(\mathcal{R}\left(x_{1}, t_{1}\right)\right)$ and $\mu_{4}\left(\mathcal{R}\left(x_{1}, t_{1}\right)\right)$, respectively. Parallel translate $\omega_{1}, \omega_{2}, \omega_{3}$ and $\omega_{4}$ along geodesics emanating from $x_{1}$ with respect to $g\left(t_{1}\right)$ to define $\omega_{1}, \omega_{2}, \omega_{3}$ and $\omega_{4}$ in a space-time neighborhood of $\left(x_{1}, t_{1}\right)$, where $\omega_{1}, \omega_{2}, \omega_{3}$ and $\omega_{4}$ are independent of time (see [3]). Then the calculation is done considering $\mathcal{R}(x, t)$ and evaluating at $\left(x_{1}, t_{1}\right)$. Moreover, by matrix analysis, for arbitrary $(x, t) \in M^{n}$, we also have (see [6])

$$
\begin{aligned}
& \mathcal{R}\left(\omega_{1}, \omega_{1}\right)+\lambda_{1} \mathcal{R}\left(\omega_{2}, \omega_{2}\right)+\lambda_{2} \mathcal{R}\left(\omega_{3}, \omega_{3}\right)+\lambda_{3} \mathcal{R}\left(\omega_{4}, \omega_{4}\right) \\
= & \inf \left\{\begin{array}{c|c}
\mathcal{R}\left(V_{i}, V_{i}\right)+\lambda_{1} \mathcal{R}\left(V_{j}, V_{j}\right) & V_{i} \perp V_{j} \perp V_{k} \perp V_{l}, 1 \leq i, j, k, l \leq N \\
+\lambda_{2} \mathcal{R}\left(V_{k}, V_{k}\right)+\lambda_{3} \mathcal{R}\left(V_{l}, V_{l}\right) & \left\|V_{i}\right\|=\left\|V_{j}\right\|=\left\|V_{k}\right\|=\left\|V_{l}\right\|=1
\end{array}\right\},
\end{aligned}
$$


where $\|\cdot\|$ denotes the Euclidean metric for the space $\wedge^{2} T^{*} M^{n}$. Then we have at $\left(x_{1}, t_{1}\right)$ :

$$
\begin{aligned}
0 \geq & \frac{\partial}{\partial t}\left(\mathcal{R}\left(\omega_{1}, \omega_{1}\right)+\lambda_{1} \mathcal{R}\left(\omega_{2}, \omega_{2}\right)+\lambda_{2} \mathcal{R}\left(\omega_{3}, \omega_{3}\right)+\lambda_{3} \mathcal{R}\left(\omega_{4}, \omega_{4}\right)\right) \\
= & \left(\frac{\partial}{\partial t} \mathcal{R}\right)\left(\omega_{1}, \omega_{1}\right)+\lambda_{1}\left(\frac{\partial}{\partial t} \mathcal{R}\right)\left(\omega_{2}, \omega_{2}\right)+\lambda_{2}\left(\frac{\partial}{\partial t} \mathcal{R}\right)\left(\omega_{3}, \omega_{3}\right) \\
& +\lambda_{3}\left(\frac{\partial}{\partial t} \mathcal{R}\right)\left(\omega_{4}, \omega_{4}\right) \\
= & \left(\Delta \mathcal{R}+\mathcal{R}^{2}+\mathcal{R}^{\#}\right)\left(\omega_{1}, \omega_{1}\right)+\lambda_{1}\left(\Delta \mathcal{R}+\mathcal{R}^{2}+\mathcal{R}^{\#}\right)\left(\omega_{2}, \omega_{2}\right) \\
& +\lambda_{2}\left(\Delta \mathcal{R}+\mathcal{R}^{2}+\mathcal{R}^{\#}\right)\left(\omega_{3}, \omega_{3}\right)+\lambda_{3}\left(\Delta \mathcal{R}+\mathcal{R}^{2}+\mathcal{R}^{\#}\right)\left(\omega_{4}, \omega_{4}\right) \\
= & \Delta\left(\mathcal{R}\left(\omega_{1}, \omega_{1}\right)+\lambda_{1} \mathcal{R}\left(\omega_{2}, \omega_{2}\right)+\lambda_{2} \mathcal{R}\left(\omega_{3}, \omega_{3}\right)+\lambda_{3} \mathcal{R}\left(\omega_{4}, \omega_{4}\right)\right) \\
& +\mu_{1}(\mathcal{R})^{2}+\lambda_{1} \mu_{2}(\mathcal{R})^{2}+\lambda_{2} \mu_{3}(\mathcal{R})^{2}+\lambda_{3} \mu_{4}(\mathcal{R})^{2} \\
& +\mathcal{R}^{\#}\left(\omega_{1}, \omega_{1}\right)+\lambda_{1} \mathcal{R}^{\#}\left(\omega_{2}, \omega_{2}\right)+\lambda_{2} \mathcal{R}^{\#}\left(\omega_{3}, \omega_{3}\right)+\lambda_{3} \mathcal{R}^{\#}\left(\omega_{4}, \omega_{4}\right) \\
\geq & \mu_{1}(\mathcal{R})^{2}+\lambda_{1} \mu_{2}(\mathcal{R})^{2}+\lambda_{2} \mu_{3}(\mathcal{R})^{2}+\lambda_{3} \mu_{4}(\mathcal{R})^{2},
\end{aligned}
$$

where the last inequality is obtained by the following two inequalities

$$
\sum_{\alpha<\beta}\left(\left(c_{1}^{\alpha \beta}\right)^{2}+\lambda_{1}\left(c_{2}^{\alpha \beta}\right)^{2}+\lambda_{2}\left(c_{3}^{\alpha \beta}\right)^{2}+\lambda_{3}\left(c_{4}^{\alpha \beta}\right)^{2}\right) \mu_{\alpha} \mu_{\beta} \geq 0
$$

and

$$
\mathcal{R}\left(\omega_{1}, \omega_{1}\right)+\lambda_{1} \mathcal{R}\left(\omega_{2}, \omega_{2}\right)+\lambda_{2} \mathcal{R}\left(\omega_{3}, \omega_{3}\right)+\lambda_{3} \mathcal{R}\left(\omega_{4}, \omega_{4}\right) \geq 0
$$

for arbitrary $x^{\prime} \neq x_{1}$, while at $\left(x_{1}, t_{1}\right)$, we have 0 . Hence

$$
\mu_{1}(\mathcal{R}(x, t))=\mu_{2}(\mathcal{R}(x, t))=\mu_{3}(\mathcal{R}(x, t))=\mu_{4}(\mathcal{R}(x, t))=0
$$

for arbitrary $(x, t) \in M^{n} \times\left[0, t_{0}\right]$, which implies that $\mathcal{R}(x, t) \geq 0$ for arbitrary $(x, t) \in M^{n} \times\left[0, t_{0}\right]$. By the maximum principle for tensors, $\mathcal{R}(x, t) \geq 0$ is preserved under the Ricci flow. Thus we have $\mathcal{R}(x, t) \geq 0$ for arbitrary $(x, t) \in M^{n} \times[0, T)$.

If (11) is satisfied, as in the proof above, we also have

$$
\mu_{1}(\mathcal{R}(x, t))=\mu_{2}(\mathcal{R}(x, t))=0
$$

for arbitrary $(x, t) \in M^{n} \times\left[0, t_{0}\right]$, which implies $\mathcal{R}(x, t) \geq 0$ for arbitrary $(x, t) \in M^{n} \times[0, T)$.

Acknowledgment. I would especially like to express my appreciation to my advisor professor Yu Zheng for longtime encouragement and meaningful discussions. I would also like to thank the referee for many suggestions. 
A NEW 3-PARAMETER CURVATURE CONDITION PRESERVED BY RICCI FLOW 845

\section{References}

[1] C. Böhm and B. Wilking, Manifolds with positive curvature operators are space forms, Ann. of Math. (2) 167 (2008), no. 3, 1079-1097.

[2] H. W. Chen, Pointwise 1/4-pinched 4-manifolds, Ann. Global Anal. Geom. 9 (1991), no. $2,161-176$

[3] B. Chow, S. C. Chu, D. Glickenstein, C. Guentheretc, J. Isenberg, T. Ivey, D. Knopf, P. Lu, F. Luo, and L. Ni, The Ricci flow: techniques and applications. Part II, Mathematical Surveys and Monographs, 144, AMS, Providence, RI, 2008.

[4] B. Chow and P. Lu, The maximum principle for systems of parabolic equations subject to an avoidance set, Pacific J. Math. 214 (2004), no. 2, 201-222.

[5] B. Chow, P. Lu, and L. Ni, Hamilton's Ricci Flow, American Mathematical Society/Science Press, Providence, 2006.

[6] R. A. Horn and C. R. Johnson, Matrix Analysis, Cambridge University Press, 1999.

[7] M. J. Micallef and J. D. Moore, Minimal two-spheres and the topology of manifolds with positive curvature on totally isotropic two-planes, Ann. of Math. (2) 127 (1988), no. 1, $199-227$.

[8] M. J. Micallef and M. Y. Wang, Metrics with nonnegative isotropic curvature, Duke Math. J. 72 (1993), no. 3, 649-672.

School of Mathematical Sciences

OCEAN UNIVERSity OF CHINA

Lane 238, SongLing Road, Laoshan District

Qingdao City, Shandong Province, 266100, P. R. China

E-mail address: gaoxiangshuli@126.com 